\title{
Controversias sobre la enfermedad tromboembólica venosa no provocada en ancianos
}

\author{
Contoversies over unprovoked venous thromboembolic disease in elderly
}

\author{
Ríos-Prego $\mathrm{M}^{1}$, Núñez-Fernández $\mathrm{MJ}^{1}$, Monreal-Bosch M² \\ ${ }^{1}$ Servicio de Medicina Interna. Complejo Hospitalario Universitario de Pontevedra. \\ ${ }^{2}$ Servicio de Medicina Interna. Hospital Universitario German Trias i Pujol. Badalona. Barcelona.
}

Desde los primeros trabajos epidemiológicos sobre la enfermedad tromboembólica venosa (ETV), que incluye la trombosis venosa profunda (TVP) y la embolia de pulmón (EP), se ha comprobado que tanto la prevalencia como (sobre todo) la incidencia de esta enfermedad aumentan a medida que lo hace la edad de la población estudiada ${ }^{1-3}$.

La estrecha relación existente entre mayor edad y mayor riesgo de ETV, se repite a lo largo de los años en distintos países (entre los que se incluye el nuestro) con condicionantes poblacionales y socio-sanitarios diferentes ${ }^{4,5}$.

Toda una serie de características hacen distinta la ETV en ancianos respecto de poblaciones de menor edad. Las manifestaciones clínicas tanto de la EP como de la TVP son diferentes en jóvenes respecto de ancianos ${ }^{6,7}$. Se confirma una mayor letalidad de la EP en ancianos ${ }^{8}$, alcanzando en este grupo de edad la $2^{\circ}$ causa de muerte en algunos estudios de necropsias ${ }^{9,10}$. En relación con el tratamiento anticoagulante mas empleado [ heparina de bajo peso molecular (HBPM) y antivitaminas $k$ (AVK)] se ha comprobado una mayor tendencia a sangrados graves, fatales e intracraneales en ancianos que en jóvenes ${ }^{11}$.

Un aspecto que transmite mayor relevancia al tema son las previsiones sobre población que el Instituto Nacional de Estadística proyecta para los próximos años en nuestro país. Se estima que los incrementos poblacionales en el período 2014-2064 en los grupos comprendidos entre los 75 años y mayores de 100 años oscila entre el $45 \%$ y el $2650 \%$. En definitiva será en los últimos segmentos de la pirámide poblacional donde se va a registrar un crecimiento más significativo para los próximos años (www.ine.es).

Los datos que se esperan para el futuro ya se están confirmando en la actualidad a nivel hospitalario. En un trabajo de Casademont et al., se evidencia un aumento progresivo de los ancianos mayores de 75 años que ingresan en los servicios de Medicina Interna de nuestro país ${ }^{12}$.

Se considera la forma no provocada 0 idiopática de ETV aquella que no presenta relación con: embarazo, puerperio, inmovilidad (médica o de causa osteoarticular sin intervención quirúrgica) cirugía (sobre todo ortopédica), viajes de más de 6 horas de duración), cáncer activo, trombofilia, 0 tratamiento hormonal ${ }^{13}$.

La forma de ETV no provocada en ancianos representa aproximadamente la mitad de todos los casos de ETV. En abril de 2014, del total de 52. 909 pacientes diagnosticados de
ETV e incluidos en el Registro Informatizado de Enfermedad Tromboembólica (RIETE), había 12. 104 pacientes con ETV no provocada, de los cuales aproximadamente el $45 \%$ eran mayores de 80 años (5. 494) (datos personales no publicados, M. J. Núñez en representación del RIETE)

Existen ciertas características que hacen diferente la forma no provocada de las formas secundarias de ETV con independencia de la edad. La primera es que el riesgo de recurrencia no se modifica con una duración de tratamiento anticoagulante superior a 3 meses $^{14}$. La segunda característica es que el riesgo de recurrencia de ETV se incrementa al suspender el tratamiento anticoagulante, siendo dicho riesgo muy superior al documentado en las formas secundarias de ETV; después de los resultados de algunos trabajos se llega a considerar que aproximadamente casi la mitad de los pacientes con una ETV no provocada, van a presentar una recurrencia en los 10 años siguientes, tras la suspensión del tratamiento anticoagulante ${ }^{15}$. Juntamente con la ETV asociada al cáncer, la forma no provocada de ETV es la que mejor define a esta patología trombótica como una enfermedad crónica con episodios de recurrencias; estos pacientes se equiparan con aquellos que tienen fibrilación auricular, por el riesgo elevado de complicaciones embólicas si no reciben tratamiento anticoagulante ${ }^{16}$.

\section{¿La ETV no provocada es cada vez "más" provocada?}

La diferenciación entre las dos categorías de ETV, provocada o no provocada, puede resultar problemática, y en ocasiones según qué condiciones consideremos, un episodio de ETV puede quedar englobado en una categoría o en otra.

En los últimos años están tomando mayor importancia dos variables que pueden hacer cambiar la clasificación de una ETV no provocada en una provocada: las infecciones y determinadas características genéticas.

Son cada vez más frecuentes las publicaciones que relacionan sin ningún lugar a dudas diferentes tipos de infecciones supuestamente banales (sobre todo respiratorias y urinarias) como factor predisponente de ETV17,18. Desde hace años está descrita la estrecha relación entre infecciones víricas por citomegalovirus y virus de la inmunodeficiencia humana, con el desarrollo de trombosis venosa ${ }^{19,20}$. De forma más reciente se están dando a conocer que patógenos bacterianos como el S. aureus y el M. tuberculosis están implicados de forma inequívoca en el desarrollo de ETV21,22. 
En trabajos sobre genética se están definiendo no sólo los genotipos específicos de ETV no provocada, si no también marcadores genéticos relacionados estrechamente con la recurrencia trombótica en la forma no provocada de ETV23,24. La aplicabilidad de las técnicas genéticas anteriormente descritas en la práctica clínica diaria, no es factible en el momento actual, y quizás tendrán que pasar años antes de que podamos disponerlas como herramientas de uso en nuestras consultas de ETV.

\section{Tratamiento de la etv no provocada en ancianos: ¿Recomendaciones de las guías?}

En la mayoría de los ensayos clínicos publicados sobre tratamiento de ETV no provocada, la edad media de los pacientes incluidos oscila entre los 53 años y los 67 años. Sólo en el estudio PROLONG, una de las ramas tenía un 74\% de pacientes con edad superior a 65 años, y una edad media de 69 años ${ }^{25}$.

Con estos datos se puede decir que el tipo de paciente incluido en la mayoría de los ensayos clínicos no son ancianos. Es lógico que tomando como base estas publicaciones, en las guías sobre tratamiento de ETV no provocada no se incluya ningún apartado específico de población anciana y pasen a ser considerados como la población general. Si seguimos las recomendaciones de las dos últimas guías de la ACCP, en pacientes (incluidos ancianos) con bajo riesgo de recurrencias y alto riesgo de sangrado el tratamiento anticoagulante debe durar sólo 3 meses; los pacientes (incluidos ancianos) con bajo-moderado riesgo de sangrado y alto riesgo de recurrencia la duración del tratamiento es indefinido, con reevaluación periódica de ambos riesgos ${ }^{26,27}$.

Cuando se aplican las reglas de predicción de sangrado (OBRI, RIETE, escala de Kuijer, escala de Kearon, HAS-BLED, HEMORRHAGES, y ATRIA) específicamente a poblaciones ancianas con ETV que reciben tratamiento anticoagulante convencional, los resultados no permiten discriminar de forma segura los grupos de alto 0 bajo riesgo de sangrado ${ }^{28,29}$. En relación con las reglas de predicción de recurrencias, no existen trabajos sobre su aplicación en ancianos, e incluso las edades medias de las personas incluidas en el estudio HERD002 (50-53 años), escala de Viena (54 años), DASH (61-62 años) y DAMOVES (61 años) no correspondan a ancianos $^{30-33}$.

En las reglas de predicción clínica de recurrencia de ETV, algunos autores proponen que sea modificado el nivel de corte del dímero-D en función de la edad. Existen diferentes puntos de corte en las cifras de dímero-D según la técnica empleada para su medición, pero lo que divide de una forma clara es la edad, según sean mayor o menor de 70 años ${ }^{34}$. Con el tratamiento indefinido se pretende disminuir de manera significativa el riesgo de recurrencia de ETV, sobre todo la EP fatal y la EP no fatal que favorece el desarrollo de hipertensión arterial pulmonar crónica. La mayoría de las publicaciones coinciden en demostrar que los pacientes con
EP previa, tienen más riesgo de que el episodio recurrente de ETV sea una nueva EP. Lo mismo sucede con los pacientes con un primer episodio de TVP, cuando recurren lo hacen generalmente en forma de TVP. Por tanto a la hora de valorar la prolongación indefinida del tratamiento anticoagulante, una de las variables a tener en cuenta es la forma de presentación inicial de ETV no provocada ${ }^{35}$.

Entre la mayoría de los expertos en el tema existe una máxima que considera la duración del tratamiento anticoagulante en la forma no provocada, como la pregunta de más difícil respuesta en el mundo de la ETV ${ }^{15}$. Con los datos presentados, en ancianos es incluso más complicado responder a esta cuestión: no podemos calcular con exactitud ni el riesgo de sangrado ni el riesgo de recurrencia, por consiguiente estimar la duración del tratamiento anticoagulante en ancianos con ETV no provocada puede ser una cuestión complicada.

\section{Anticoagulantes orales de acción directa: Experiencia en ancianos con ETV no provocada}

Disponemos de datos sobre la utilización de los anticoagulantes orales de acción directa (AOAD) en el tratamiento de la ETV, tanto en los primeros 3 meses, como en la fase de tratamiento prolongado que comprende el período posterior a los 3 meses iniciales.

En los primeros tres meses, el tratamiento con $A O A D$ en la ETV es igual de efectivo y más seguro que el tratamiento convencional con heparina de bajo peso molecular y antivitaminas K. Estas conclusiones se obtienen de diferentes meta-análisis cuya base son los ensayos clínicos RE-COVER I y RE-COVER II (con dabigatran), EISNTEIN-DVT y EINSTEIN-EP (con rivaroxaban), AMPLIFY (con apixaban) y HOKUSAI (con edoxaban) ${ }^{36}$.

Sin embargo en dichos ensayos, el porcentaje de pacientes mayores de 75 años es minoritario, oscila entre el $9 \%$ y el $16 \%$, y no se especifican los porcentajes de ETV no provocada $^{36}$.

En los ensayos clínicos de tratamiento anticoagulante prolongado con duración superior a tres meses, el porcentaje de pacientes con ETV no provocada es muy elevado, entre el $70 \%$ y el $93 \%$. Sin embargo al analizar las edades, nos encontramos en la misma situación de los ensayos de tratamiento inferior a 3 meses: la mayoría de los pacientes incluidos en dichos ensayos, no se pueden considerar ancianos; la media de edad oscila entre los 55 y los 58 años ${ }^{37-40}$. Los estudios RE-SONATE (con dabigatran), EINSTEIN-EXT (con rivaroxaban), y AMPLIFY-EXT (con apixaban), comparan AOAD frente placebo; sólo en el RE-MEDY, existe una comparación de dabigatran frente tratamiento convencional. Los diferentes meta-análisis publicados sobre tratamiento prolongado en ETV donde se comparan diferentes opciones, Ios AOAD presentan una eficacia igual al tratamiento convencional, con menor porcentaje de sangrados mayores y recurrencias de trombosis ${ }^{37-40}$. Se documenta el sangrado 
mayor en 0'56 por 100 pacientes/año (IC: 0'24-1'1) de los tratados con $A O A D$, frente el 1 ' 6 por 100 pacientes años (IC: 1'2-2'1) de los que reciben tratamiento con AVK. No hay evidencia de sangrados fatales en los ensayos clínicos con $A O A D$, frente un $6{ }^{\prime} 8 \%$ de letalidad de los pacientes tratados con AVK ${ }^{39}$.

El efecto secundario más temido del tratamiento con AVK son los sangrados. Numerosas publicaciones demuestran que los ancianos de más de 75 años son un grupo más vulnerable, al presentar una mayor proporción de sangrados menores, mayores y sobre todo, sangrados intracraneales, respecto de la población con menos de 75 años ${ }^{41-44}$.

La hemorragia intracraneal espontánea (HICE) no es la más frecuente de las complicaciones hemorrágicas asociadas a tratamiento con AVK, pero sí la más temida por su alta mortalidad (oscila entre $33 \%$ y $50 \%$ ) y su gran morbilidad que condiciona en los supervivientes importantes secuelas neurológicas ${ }^{45,46}$. Los principales datos sobre riesgo de HICE asociada a AOAD se disponen a partir de comparaciones indirectas de los estudios pivotales en fibrilación auricular ${ }^{47-49}$. Es muy significativo que en población anciana (mayores de 75 y 80 años), tanto dabigatran, como rivaroxaban, apixaban y edoxaban, presenten menor porcentaje de HICE respecto al tratamiento convencional con AVK.

Datos disponibles en el mundo real sobre la utilización de AOAD, los podemos extraer de registros como el RIETE, donde se incluyen pacientes diagnosticados de ETV y en tratamiento anticoagulante. En un trabajo realizado hace un año con datos procedentes del RIETE, se incluyeron 3. 963 pacientes, entre 66 y 80 años y 2241 pacientes mayores de 80 años, con ETV no provocada; la utilización de AOAD en el primer grupo fue de 0'98\% de pacientes en la fase aguda, y del 3'5\% después del tercer mes; mientras que en el grupo de más de 80 años, los AOAD se utilizaron en la fase aguda en el 0'45\% de pacientes, documentándose su uso en $1^{\prime} 7 \%$ de pacientes con posterioridad al tercer mes (datos personales no publicados, M. J. Núñez en representación del RIETE ).

En definitiva, a pesar de que los AOAD presentan un gran potencial en ancianos, respecto de la anticoagulación convencional, su utilización en personas de más de 75 años está muy por debajo de lo esperado.

\section{Perspectivas inmediatas y futuras}

En los próximos meses irán apareciendo publicaciones que tienen como base pacientes con ETV no provocada. Ya se han terminado de recoger los datos del estudio TEVIS, un estudio multicéntrico retrospectivo, llevado a cabo en nuestro país, en el que han participado gran cantidad de hospitales, incluidos los de nuestra Comunidad Autónoma.

Está en marcha el estudio prospectivo ExACT, que puede arrojar más luz sobre el tema de la ETV no provocada, también en ancianos, con la pretensión de ver la evolución de varias cohortes de pacientes con y sin tratamiento anticoa- gulante, comparando eventos como recurrencia de ETV y sangrados durante dos años ${ }^{50}$.

En nuestra comunidad autónoma, se está llevando a cabo un estudio prospectivo sobre ETV no provocada, con inclusión de ancianos, dirigido por los Drs. Monte y Pombo, en la que participan la mayoría de los hospitales gallegos; se espera que en un plazo de unos meses se conozca la situación de esta patología trombótica en nuestro entorno más cercano. Será difícil conseguir que en algún momento se pueda llevar a cabo un estudio sobre la ETV no provocada con las premisas que recomienda M. Rodger: estudio multicéntrico, que englobe múltiples países, con una muestra muy amplia con miles de pacientes, randomizando en dos grupos (tratamiento finalizado frente tratamiento indefinido), seguidos durante más de 10 años, estudiando eventos (recurrencias y sangrados en ambos grupos) y todas las causas de mortalidad'5.

Lo más cercano a estas condiciones casi utópicas lo podemos encontrar en el RIETE. Es deseable que en un futuro no muy lejano, se publiquen datos al respecto extraídos desde dicho registro y que abarque específicamente población anciana con más de 80 años. Será interesante comparar los sangrados letales durante el tratamiento anticoagulante, frente las recurrencias letales en el período siguiente a la suspensión del mismo. Se podrá responder si se puede manejar de la misma forma el riesgo de recurrencia en una EP que en una TVP.

Con el paso del tiempo esperamos tener más datos en el mundo real sobre la utilización de los nuevos $A O A D$ en el tratamiento agudo de la ETV en ancianos, pero sobre todo nos interesa en la fase de tratamiento prolongado, con el objetivo de confirmar las excelentes perspectivas que en este apartado presentan los AOAD.

\section{Bibliografía}

1. Siccama R. N., Janssen K. J., Verheijden N. A., Oudega R., Bax L., van Delden J. J., Moons K. G. Systematic review: diagnostic accuracy of clinical decision rules for venous thromboembolism in elderly. Ageing Res Rev. 2011; 10: 304-13.

2. Anderson F. A. Jr., Wheeler H. B., Goldberg R. J., Hosmer D. W., Patwardhan N. A., Jovanovic B., Forcier A., Dalen J. E. A population-based perspective of the hospital incidence and case-fatality rates of deep vein thrombosis and pulmonary embolism. The Worcester DVT Study. Arch Intern Med. 1991; 151:933-8.

3. Silverstein M. D., Heit J. A., Mohr D. N., Petterson T. M., O'Fallon W. M., Melton III L. J. Trends in the Incidence of Deep Vein Thrombosis and Pulmonary EmbolismA 25-Year Population-Based StudyArch Intern Med. 1998;158:585-593.

4. Naess I. A., Christiansen S. C., Romundstad P., Cannegieter S. C., Rosendaal F. R., Hammerstrøm J. Incidence and mortality of venous thrombosis: a population-based study. J Thromb Haemost. 2007; 5 :692-9.

5. Guijarro Merino R., Montes Santiago J., San Román Terán C. M. Epidemiología hosPitalaria de la enfermedad tromboembólica venosa en España. Med Clin (Barc). 2008 ;131 Suppl 2:2-9

6. Kokturk N., Oguzulgen I. K., Demir N., Demirel K., Ekim N. Differences in clinical presentation of pulmonary embolism in older vs younger patients. Circ J. 2005; $69: 981-6$.

7. Le Gal G., Righini M., Roy P. M., Meyer G., Aujesky D., Perrier A., Bounameaux H. Differential value of risk factors and clinical signs for diagnosing pulmonary embolism according to age. J Thromb Haemost. 2005; 3:2457-64.

8. Stein P. D., Matta F., Alrifai A. Case fatality rate in pulmonary embolism according to age and stability. Clin Appl Thromb Hemost. 2013; 19: 668-72

9. Gee WM. Causes of death in a hospitalized geriatric population: an autopsy study of 3000 patients. Virchows Arch A Pathol Anat Histopathol. 1993; 423: 343-9.

10. Berzlanovich A. M., Missliwetz J., Sim E., Fazeny-Dörner B., Fasching P., Marosi C., Waldhoer T., Muhm M. Unexpected out-of-hospital deaths in persons aged 85 years or older: an autopsy study of 1886 patients. Am J Med 2003; 114:365-9. 
11. Palareti G., Cosmi B. Bleeding with anticoagulation therapy - who is at risk, and how best to identify such patients. Thromb Haemost. 2009; 102: 268-78.

12. Casademont J., Francia E., Torres 0 . La edad de los pacientes atendidos en los servicios de medicina interna en España: una perspectiva de 20 años. Med Clin (Barcelona); 2012: 138: 289-292

13. Prandoni P., Noventa F., Ghirarduzzi A., Pengo V., Bernardi E., Pesavento R., lotti M., Tormene D., Simioni P., Pagnan A.

The risk of recurrent venous thromboembolism after discoontinuing anticoagulation in patients with acute proximal deep vein thrombosis or pulmonary embolism. A prospective cohort study in 1626 patients. Haematologica. 2007; 92:199-205.

14. Boutitie F., Laurent Pinede L., Schulman S., Agnelli G., Raskob G., Julian J.,Hirsh J., Kearon $\mathrm{C}$. Influence of preceding length of anticoagulant treatment and initial presentation of venous thromboembolism on risk of recurrence after stopping treatment: analysis of individual participants' data from seven trials. BMJ 2011;342:d3036

15. Rodger M., Carrier M., Gandara E., Le Gal G. Unprovoked venous thromboembolism: Short term or indefinite anticoagulation? Balancing long-term risk and benefit. Blood Rev. 2010; 24: 171-8

16. Heit J. A., Lahr B. D., Ashrani A. A., Petterson T. M., Bailey K. R. Predictors of venous thromboembolism recurrence, adjusted for treatments and interim exposures: a population-based case-cohort study. Thromb Res. 2015; 136: 298-307

17. Smeeth L., Cook C., Thomas S., Hall A. J., Hubbard R., Vallance P. Risk of deep vein thrombosis and pulmonary embolism after acute infection in a community setting. Lancet. 2006; 367(9516):1075-9.

18. Schmidt M., Horvath-Puho E, Thomsen RW, Smeeth L, Sørensen HT. Acute infections and venous thromboembolism. J Intern Med 2012; 271: 608-618.

19. Paran Y., Shalev V., Steinvil A., Justo D., Zimmerman O., Finn T., Berliner S., Zeltser D., Weitzman D., Raz R., Chodick G. Thrombosis following acute cytomegalovirus infection: a community prospective study. Ann Hematol 2013; 92: 969-974.

20. Auerbach E., Aboulafia D. M. Venous and arterial thromboembolic complications associated with HIV infection and highly active antiretroviral therapy. Semin Thromb Hemost. 2012; 38: 830-8.

21. Mejer N., Westh H., Schonheyder H. C., Jensen A. G., Larsen A. R., Skov R., Benfield T.,Danish Staphylococcal Bacteraemia Study Group. Increased risk of venous thromboembolism within the first year after Staphylococcus aureusbacteraemia: a nationwide observational matched cohort study. J Intern Med 2014; 275: 387-397.

22. Dentan C., Epaulard O., Seynaeve D., Genty C., Bosson J. L.

Active tuberculosis and venous thromboembolism: association according to international classification of diseases, ninth revision hospital discharge diagnosis codes. Clin Infect Dis 2014; 58: 495-501.

23. Lewis D. A., Suchindran S., Beckman M. G., Hooper W. C., Grant A. M., Heit J. A.,Manco-Johnson M., Moll S., Philipp C. S., Kenney K., De Staercke C., Pyle M. E., Chi J. T., Ortel T. L. Whole blood gene expression profiles distinguish clinical phenotypes of venous thromboembolism. Thromb Res. 2015; 135: 659-65.

24. Montes R, Guruceaga E, González-Porras JR, Reverter JC, Marco P, Pina E, Páramo JA, Hermida J, Lecumberri R; Grupo de Trabajo de Patología Trombótica de la Sociedad Española de Trombosis y Hemostasia (SETH). Identification of new markers of recurrence in patients with unprovoked deep vein thrombosis by gene expression profiling: the retro study. Eur J Haematol. 20152015 Oct 27. doi: 10. 1111/ejh. 12692.

25. Kearon C. Indefinite anticoagulation after a first episode of unprovoked venous thromboembolism: yes. J Thromb Haemost. 2007; 5: 2330-5

26. Kearon C., Akl E. A., Comerota A. J., Prandoni P., Bounameaux H., Goldhaber S. Z., Nelson M. E., Wells P. S., Gould M. K., Dentali F., Crowther M., Kahn S. R. ; American College of Chest Physicians. Antithrombotic therapy for VTE disease: Antithrombotic Therapy and Prevention of Thrombosis, 9th ed: American College of Chest Physicians Evidence-Based Clinical Practice Guidelines. Chest. 2012; 141 (2 Suppl):e419S-94S.

27. Kearon C., Akl E. A., Ornelas J., Blaivas A., Jiménez D., Bounameux H., Huisman M., King C. S., Morris T. A., Sood N., Stevens S. M., Vintch J. R., Wells P., Woller S. C., Moores L. Antithrombotic Therapy for VTE Disease: CHEST Guideline and Expert Panel Report. Chest 2016; 149: 315-52.

28. Scherz N., Méan M., Limacher A., Righini M., Jaeger K., Beer H. J., Frauchinger B.,Osterwalder J., Kucher N., Matter C. M., Banyai M., Angelillo-Scherrer A., Lämmle B., Husmann M., Egloff M., Aschwanden M., Bounameaux H., Cornuz J., Rondini N., Aujesky D. Prospective, multicenter validation of prediction scores for major bleeding in elderly patients with venous thromboembolism. J Thromb Haemost 2013; 11: 435-443.

29. Poli D., Antonucci E., Testa S., Cosmi B., Palareti G., Ageno W. ; FCSA Italian Federation of Anticoagulation Clinics. The predictive ability of bleeding risk stratification models in very old patients on vitamin $\mathrm{K}$ antagonist treatment for venous thromboembolism: results of the prospective collaborative EPICA study. J Thromb Haemost. 2013; 11:1053-8

30. Rodger M. A., Kahn S. R., Wells P. S., Anderson D. A., Chagnon I., Le Gal G., Solymoss S., Crowther M., Perrier A., White R., Vickars L., Ramsay T., Betancourt M. T., Kovacs M. J. Identifying unprovoked thromboembolism patients at low risk for recurrence who can discontinue anticoagulant therapy. CMAJ. 2008; 179: 417-26
31. Eichinger S., Heinze G., Jandeck L. M., Kyrle P. A. Risk assessment of recurrence in patients with unprovoked deep vein thrombosis or pulmonary embolism: the Vienna prediction model. Circulation. 2010; 121: 1630-6

32. Tosetto A., lorio A., Marcucci M., Baglin T., Cushman M., Eichinger S., Palareti G., Poli D., Tait R. C., Douketis J. Predicting disease recurrence in patients with previous unprovoked venous thromboembolism: a proposed prediction score (DASH). J Thromb Haemost. 2012; 10: 1019-25.

33. Fraco Moreno A. I., García Navarro M. J., Ortiz Sánchez J., Martín Díaz R. M., Madroñal Cerezo E., de Ancos Araxil C. L., Cabello Clotet N., Perales Fraile I., Giemeno García S., Montero Hernández C., Zapatero Gaviria A., Ruiz Giardín J. M. A risk score for prediction of recurrence in patients with unprovoked venous thromboembolism (DAMOVES). Eur J Intern Med 2016; Jan 7. pii: S0953-6205(15)00438-0. doi: 10. 1016/j. ejim. 2015. 12. 010.

34. Palareti G., Cosmi B., Legnani C., Antonucci E., De Micheli V., Ghirarduzzi A., Poli D., Testa S., Tosetto A., Pengo V., Prandoni P. ; DULCIS (D-dimer and ULtrasonography in Combination Italian Study) Investigators. D-dimer to guide the duration of anticoagulation in patients with venous thromboembolism: a management study. Blood. 2014; 124: 196-203

35. Baglin T., Douketis J., Tosetto A., Marcucci M., Cushman M., Kyrle P., Palareti G., Poli D., Tait R. C., lorio A. Does the clinical presentation and extent of venous thrombosis predict likelihood and type of recurrence? A patient-level meta-analysis. J Thromb Haemost. 2010 Nov;8(11):2436-42.

36. Gómez-Outes A, Terleira-Fernández Al, Lecumberri R, Suárez-Gea ML, Vargas-Castrillón E. Direct oral anticoagulants in the treatment of acute venous thromboembolism: a systematic review and meta-analysis. Thromb Res. 2014; 134: 774-82.

37. Castellucci L. A., Cameron C., Le Gal G., Rodger M. A., Coyle D., Wells P. S, Clifford T.,Gandara E., Wells G., Carrier M. Efficacy and safety outcomes of oral anticoagulants and antiplatelet drugs in the secondary prevention of venous thromboembolism: systematic review and network meta-analysis. BMJ 2013; 347: f5133.

38. Gómez-Outes A., Lecumberri R., Suárez-Gea M. L., Terleira-Fernández A. I., Monreal M., Vargas-Castrillón E. Case Fatality Rates of Recurrent Thromboembolism and Bleeding in Patients Receiving Direct Oral Anticoagulants for the Initial and Extended Treatment of Venous Thromboembolism: A Systematic Review. J Cardiovasc Pharmacol Ther. 2015; 20: 490-500.

39. Wu C., Alotaibi G. S., Alsaleh K., Linkins L. A., McMurtry M. S. Case-fatality of recurrent venous thromboembolism and major bleeding associated with aspirin, warfarin, and direct oral anticoagulants for secondary prevention. Thromb Res 2015; 135: 243-248.

40. Marik P. E., Cavallazzi R. Extended Anticoagulant and Aspirin Treatment for the Secondary Prevention of Thromboembolic Disease: A Systematic Review and Meta-Analysis. PLoS One. 2015 Nov 20;10(11):e0143252.

41. van der Meer F. J., Rosendaal F. R., Vandenbroucke J. P., Briët E. Bleeding complications in oral anticoagulant therapy. An analysis of risk factors. Arch Intern Med. 1993; 153: 1557-62.

42. Palareti G., Hirsh J., Legnani C., Manotti C., D’Angelo A., Pengo V., Moia M., Guazzaloca G., Musolesi S., Coccheri S. Oral anticoagulation treatment in the elderly: a nested, prospective, case control study. Arch Intern Med. 2000; 160: 470-8.

43. Fang M. C., Go A. S., Hylek E. M., Chang Y., Henault L. E., Jensvold N. G., Singer D. E. Age and the risk of warfarin-associated hemorrhage: the anticoagulation and risk factors in atrial fibrillation study. J Am Geriatr Soc. 2006; 54: 1231-6.

44. Hylek E. M., Evans-Molina C., Shea C., Henault L. E., Regan S. Major hemorrhage and tolerability of warfarin in the first year of therapy among elderly patients with atrial fibrillation. Circulation. 2007; 115: 2689-96.

45. Fang M. C., Go A. S., Chang Y., Hylek E. M., Henault L. E., Jensvold N. G., Singer D. E. Death and disability from warfarin-associated intracranial and extracranial hemorrhages. Am J Med. 2007; 120: 700-5.

46. Cervera A., Amaro S., Chamorro A. Oral anticoagulant-associated intracerebral hemorrhage. J Neurol. 2012 Feb;259(2):212-24.

47. Baraco S., Cheung Y. W., Eikelboom J. W., Coppens M. New oral anticoagulants in elderly patients. Best Pract Res Clin Haematol 2013; 26: 215-224

48. Yates S. W. Novel oral anticoagulants for stroke prevention in atrial fibrillation: a focus on the older patient. Int J Gen Med. 2013; 6: 167-80.

49. Potpara T. S., Lip GY. Novel oral anticoagulants in non-valvular atrial fibrillation. Best Pract Res Clin Haematol. 2013; 26: 115-29

50. Tullett J, Murray E, Nichols L, Holder R, Lester W, Rose P, Hobbs FD, Fitzmaurice D. Trial Protocol: a randomised controlled trial of extended anticoagulation treatment versus routine anticoagulation treatment for the prevention of recurrent VTE and post thrombotic syndrome in patients being treated for a first episode of unprovoked VTE (The ExACT Study). BMC Cardiovasc Disord. 2013 Mar 9;13:16. 